\title{
Prophylactic Peritoneal Drainage is Associated with Improved Fluid Output after Congenital Heart Surgery
}

\author{
Kevin A. Pettit ${ }^{1}$ - Nicholas A. Schreiter ${ }^{2}$ - Entela B. Lushaj ${ }^{3}$. Joshua L. Hermsen ${ }^{3}$ - Michael Wilhelm ${ }^{1}$. \\ Allison C. Redpath Mahon ${ }^{1} \cdot$ Kari L. Nelson ${ }^{3}$. Joshua J. DeGrave ${ }^{4} \cdot$ Nicholas Marka $^{3}$. Petros V. Anagnostopoulos ${ }^{3,5}$
}

Received: 31 May 2020 / Accepted: 22 July 2020 / Published online: 30 July 2020

(c) Springer Science+Business Media, LLC, part of Springer Nature 2020

\begin{abstract}
Infants undergoing congenital heart surgery (CHS) with cardiopulmonary bypass (CPB) are at risk of acute kidney injury (AKI) and fluid overload. We hypothesized that placement of a passive peritoneal drain (PPD) can improve postoperative fluid output in such infants. We analyzed 115 consecutive patients, age birth to 60 days, admitted to the PICU after CHS with CPB between 2012 and 2018. Patients who needed postoperative ECMO were excluded. Linear and logistic regression models compared postoperative fluid balances, diuretics administration, AKI, vasoactive-inotropic scores (VIS), time intubated, and length of stay after adjusting for pre/operative predictors including STAT category, bypass time, age, weight, and open chest status. PPD patients had higher STAT category $(p=0.001)$, longer CPB times $(p=0.001)$, and higher VIS on POD 1-3 ( $p \leq 0.005$ daily). PPD patients also had higher AKI rates $(p=0.01)$ that did not reach significance in multivariable modeling. There were no postoperative deaths. Postoperative hours of intubation, hospital length of stay, and POD 1-5 fluid intake did not differ between groups. Over POD 1-5, PPD use accounted for $48.8 \mathrm{~mL} / \mathrm{kg}$ increased fluid output (95\% CI $[2.2,95.4], p=0.043)$ and $3.41 \mathrm{mg} / \mathrm{kg}$ less furosemide administered (95\% CI [1.69, 5.14], $p<0.001)$. No PPD complications were observed. Although PPD placement did not affect end-outcomes, it was used in higher acuity patients. PPD placement is associated with improved fluid output despite lower diuretic administration and may be a useful postoperative fluid management adjunct in some complex CHS patients.
\end{abstract}

Keywords Perioperative care $\cdot$ Congenital heart surgery $\cdot$ Neonate $\cdot$ Postoperative care

Petros V. Anagnostopoulos

petros@surgery.wisc.edu

1 Department of Pediatrics, The American Family Children's Hospital, University of Wisconsin Hospital and Clinics, 1675 Highland Ave, Madison, WI 53792, USA

2 School of Medicine and Public Health, The American Family Children's Hospital, University of Wisconsin Hospital and Clinics, 1675 Highland Ave, Madison, WI 53792, USA

3 Division of Cardiothoracic Surgery, Department of Surgery, The American Family Children's Hospital, University of Wisconsin Hospital and Clinics, 1675 Highland Ave, Madison, WI 53792, USA

4 School of Pharmacy, The American Family Children's Hospital, University of Wisconsin Hospital and Clinics, 1675 Highland Ave, Madison, WI 53792, USA

5 University of Wisconsin Department of Surgery Clinical Science Center, 600 Highland Ave, Madison, WI 53792, USA

\section{Introduction}

Infants undergoing congenital heart surgery (CHS) with cardiopulmonary bypass are at high risk of developing acute kidney injury (AKI) and fluid overload (FO). Infants receive proportionally more fluid than older patients relative to their size, increasing their risk of FO. Fluid overload, independent of AKI, is associated with increased length of stay and mortality in neonates undergoing heart surgery [1]. Interestingly, FO in these patients is also associated with worse AKI, likely for multifactorial reasons [1, 2]. Some institutions routinely place peritoneal catheters to manage postoperative FO in neonates and young infants, using either passive peritoneal drainage, peritoneal dialysis, or both [3-8].

When used for dialysis after cardiac surgery, peritoneal catheters pose a low risk to the patient and have demonstrated benefits including better fluid management, quicker time to reach net-negative fluid balance, reduction in intraabdominal hypertension, and even reduced serum markers 
of inflammation [3-6]. It is unclear whether the placement of passive peritoneal drains (PPDs) carry similar benefits, as small studies with dissimilar cohorts have yielded disparate results across different measures [7-9].

In our center, we place PPDs in select young infants (under 60 days) undergoing CHS who are deemed at greater risk of AKI and FO based on preoperative assessment or who are at high risk of postoperative diastolic dysfunction. Passive drainage begins in the immediate postoperative period in the Pediatric Intensive Care Unit, and drains are removed as the drain output downtrends and urine output has recovered. We hypothesized that prophylactic passive peritoneal drainage improves postoperative fluid output in these infants, specifically when controlling for the greater preoperative risk profile of the patients in whom they are placed. The aim of this retrospective study was to assess the effect of PPD placement on fluid management in these patients.

\section{Material and Methods}

This project was approved by the University of Wisconsin Institutional Review Board with patient consent waived. Patients for this retrospective cohort study were identified through the Society of Thoracic Surgeons (STS) database. STS data and retrospective chart review data were collected for all patients under 60 days of age who underwent congenital heart surgery (CHS) requiring cardiopulmonary bypass at the American Family Children's Hospital between January 2012 and September 2018. A diverse set of lesions were represented (Table 1). Patients who required ECMO over their postoperative hospital course were excluded from analysis. Preoperative data included weight, creatinine, presence of chromosomal abnormality or syndrome, age at surgery, sex, history of low birth weight, and history of premature birth. Operative data included cardiopulmonary bypass time, aortic cross clamp time, Society of Thoracic Surgeons-European Association for Cardio-Thoracic Surgery (STAT) category, emergence from the operating room with open chest, and prophylactic insertion of a PPD.

Intraoperatively, modified ultrafiltration was used on all patients for this study. PPD placement occurred in the operating room following reversal of heparin. Patients were not randomized and were selected for PPD placement proactively by the surgical team out of concern for preoperative risk factors that may increase risk of postoperative fluid overload, such as higher STAT score, risk of diastolic dysfunction, and younger age. Single-cuff, $31 \mathrm{~cm}$ straight Tenckhoff peritoneal catheters (Covidien, Minneapolis, MN) were placed in the epigastrium and directed to the left pericolic gutter under direct visualization. The peritoneum was closed separately from the drain entrance site. To reduce the possibility of incisional hernia, a 5-0 polypropylene stay
Table 1 PPD and non-PPD cohorts had a broad array of diagnoses

\begin{tabular}{lll}
\hline Lesion & $\begin{array}{l}\text { PPD }(n=34) \\
n(\%)\end{array}$ & $\begin{array}{l}\text { No PPD }(n=81) \\
n(\%)\end{array}$ \\
\hline VSD & $1(2.9)$ & $1(1.2)$ \\
Single ventricle & $11(32.3)$ & $4(4.9)$ \\
AV canal defects & $0(0)$ & $3(3.7)$ \\
Cor triatrium & $0(0)$ & $3(3.7)$ \\
Tetralogy of fallot & $1(2.9)$ & $9(11.1)$ \\
Coronary anomaly & $1(2.9)$ & $1(1.2)$ \\
D-TGA & $6(17.6)$ & $13(16.0)$ \\
D-TGA and VSD & $2(5.9)$ & $2(2.5)$ \\
CoAo & $0(0)$ & $2(2.5)$ \\
CoAo with other lesions & $1(2.9)$ & $8(9.9)$ \\
Aortic Ah hyplasia & $3(8.8)$ & $13(16.0)$ \\
IAA & $3(8.8)$ & $0(0)$ \\
IAA and VSD & $1(2.9)$ & $2(2.5)$ \\
Hemitruncus arteriosus & $0(0)$ & $1(1.2)$ \\
Truncus arterius & $2(5.9)$ & $2(2.5)$ \\
TAPVC & $2(5.9)$ & $8(9.9)$ \\
Ebstein anoly & $0(0)$ & $1(1.2)$ \\
DORV & $0(0)$ & $5(6.2)$ \\
Other lesions & $0(0)$ & $3(3.7)$ \\
\hline
\end{tabular}

$A V$ Atrioventricular, CoAo coarctation of the aorta, DILV double inlet left ventricle, $D O R V$ double outlet right ventricle, $D-T G A$ dextrotransposition of the great arteries, HLHS hypoplastic left heart syndrome, IAA interrupted aortic arch, TAPVC total anomalous pulmonary venous connection, $V S D$ ventricular septal defect

suture was pre-placed through the abdominal wall at the stab incision site. The collection bag was connected and kept on the bed. Drains were removed while patients were still intubated and as urine output increased, although there was no absolute volume threshold or protocol for drain removal. The surgical team and approaches remained the same throughout the timeframe examined in this study, and there was no era differentiation. Postoperative care pathways and institutional practices for the management of postoperative CHS patients did not change through the course of the study. Notably, dialysate was not used for these patients; our institution rarely uses peritoneal dialysis postoperatively but has in the case of significant AKI with hyperkalemia, which was not experienced by patients in this cohort.

Postoperative data analyzed included daily weight (in kilograms), urine output (mL/kg/24 h), PPD output (mL/ $\mathrm{kg} / 24 \mathrm{~h}$ ), total fluid output $(\mathrm{mL} / \mathrm{kg} / 24 \mathrm{~h})$, and total fluid intake $(\mathrm{mL} / \mathrm{kg} / 24 \mathrm{~h})$ for 5 days postoperatively or two days after removal of the PPD, whichever came last. Total fluid output included chest and mediastinal drain output. The incidence of AKI within the first ten postoperative days, as determined by the creatinine component of the neonatal modified Kidney Disease: Improving Global Outcomes (KDIGO) criteria, was determined [10, 11]. KDIGO urine 
output criteria was not utilized given that it was anticipated that PPD placement would likely be associated with lower urine output. First day with net negative fluid balance was recorded. Daily furosemide dosing per preoperative kilogram weight was tracked over POD 1-5. Chlorothiazide, spironolactone, bumetanide, and hydrochlorothiazide administration were also tracked.

We examined several variables to detect physiologic consequences of postoperative fluid overload, including time to chest closure for those with delayed sternal closure, duration of postoperative invasive ventilation (hours), immediate postoperative dynamic respiratory compliance, and daily dynamic respiratory compliance at $7 \mathrm{am}$. The dynamic respiratory compliance examined was a single value within 60 min of 7 am and the closest value to that time. Additionally, maximum daily vasoactive-inotropic score was calculated through POD 3 [12]. Death within 30 days of surgery, death prior to discharge, STS surgical mortality, and hospital length of stay were noted. Of note, our institution uses an adjustable care model in which the patient stays in the same ICU room until discharge, preventing measurement of ICU length of stay. Total daily diuretic administration was calculated through POD 5.

\section{Statistical methods}

All analyses were conducted using $R$ software version 3.6.0 (Vienna, Austria). For univariate analysis, continuous variables were reported as mean, standard deviation, and $t$ test $p$ value. Preoperative and operative input data were compared between PPD and non-PPD groups, and multivariable analysis was completed for inputs with between-group $\mathrm{p}$ values of $\leq 0.1$. Ultimately, models utilized each of the following predictors: STAT category, bypass time, age at surgery, weight at surgery, and presence of delayed chest closure (Table 2).

Multivariable outcomes of interest included fluid intake $(\mathrm{mL} / \mathrm{kd})$, urine output $(\mathrm{mL} / \mathrm{kg})$, total fluid output $(\mathrm{mL} / \mathrm{kg})$, and furosemide administration $(\mathrm{mg} / \mathrm{kg}$ ), each cumulatively over POD 1-5. Other multivariable outcomes of interest included POD 0 fluid intake $(\mathrm{mL} / \mathrm{kg})$, daily percent increase above preoperative weight for POD $1-5$, number of postoperative days to first day net negative, incidence of KDIGO Stage 1 and 2 AKI by creatinine criteria, and peak daily vasoactive-inotropic score (VIS) over POD 0-3. Postoperative hours on ventilator, postoperative days to delayed chest closure in the presence of an open chest, postoperative hospital length of stay, dynamic respiratory compliance $(\mathrm{mL} / \mathrm{cmH} 2 \mathrm{O})$ on postoperative arrival to the pediatric ICU, and dynamic respiratory compliance $(\mathrm{mL} / \mathrm{cmH} 2 \mathrm{O})$ at $7 \mathrm{am}$ POD1-2 were also included in multivariable analysis.

In multivariable modelling, STAT category was treated as a categorical predicator with a baseline of 1 . For continuous outcomes, a multivariable linear regression model was fit to adjust for the above predictors. Results were reported as effect estimate, $95 \%$ confidence interval for the effect estimate, and the $p$ value testing whether the effect estimate is significantly different from 0 , with a significance threshold set a priori as $p \leq 0.05$. All outcomes measured were continuous except for incidence of KGIDO creatinine stage $1 \mathrm{AKI}$ and incidence of KDIGO creatinine stage 2 AKI, which were categorical. For these categorical outcomes, a
Table 2 Preoperative and operative patient demographics demonstrate that the PPD patient cohort has a higher risk profile with significant differences in STAT category, bypass time, age at surgery, and prevalence of delayed chest closure

\begin{tabular}{|c|c|c|c|}
\hline & $\begin{array}{l}\mathrm{PPD}(n=34) \\
n(\mathrm{SD} \text { or } \% \text { sample })\end{array}$ & $\begin{array}{l}\text { No PPD }(n=81) \\
n(\mathrm{SD} \text { or } \% \text { sample })\end{array}$ & $P$-value \\
\hline Female sex & $12(35 \%)$ & $34(42 \%)$ & 0.539 \\
\hline Premature birth ( $<36$ weeks) & $4(12 \%)$ & $18(22 \%)$ & 0.298 \\
\hline Identified syndrome or chromosomal disorder & $8(24 \%)$ & $19(24 \%)$ & 1 \\
\hline Low birth weight $(<2 \mathrm{~kg})$ & $2(6 \%)$ & $8(10 \%)$ & 0.721 \\
\hline Presence of delayed chest closure & $25(74 \%)$ & $18(22 \%)$ & $<0.001$ \\
\hline Weight at surgery (kg) & $3.20(0.53)$ & $3.42(0.77)$ & 0.09 \\
\hline Preoperative creatinine & $0.50(0.17)$ & $0.44(0.14)$ & 0.114 \\
\hline Age at surgery (days) & $10.56(12.64)$ & $16.49(15.85)$ & 0.037 \\
\hline Bypass time (minutes) & $185.62(46.23)$ & $152.74(45.27)$ & 0.001 \\
\hline Cross clamp time (minutes) & $82.62(29.91)$ & $72.59(34.22)$ & 0.141 \\
\hline STAT category 1 & $1(3 \%)$ & $2(2 \%)$ & $<0.001$ \\
\hline STAT category 2 & $2(6 \%)$ & $13(16 \%)$ & \\
\hline STAT category 3 & $6(18 \%)$ & $15(19 \%)$ & \\
\hline STAT category 4 & $12(35 \%)$ & $47(58 \%)$ & \\
\hline STAT category 5 & $13(38 \%)$ & $4(5 \%)$ & \\
\hline
\end{tabular}

OR Operating room, $P P D$ passive peritoneal drain, $S D$ standard deviation, STAT society of thoracic surgeons-European Association for Cardio-Thoracic Surgery 
multivariable logistic regression model was fit adjusting for the aforementioned predictors. Results were reported with an odds ratio, 95\% confidence for the odds ratio, and $p$ value testing whether the odds ratio is significantly different from 1 , with a significance threshold set a priori as $p \leq 0.05$.

\section{Results}

Between January 2012 and September 2018, 124 CHS cases requiring bypass were performed in children under 60 days. Of these patients, 9 were excluded from analysis for requiring postoperative ECMO (4 PPD, 5 no PPD), leaving for analysis 34 patients who received prophylactic PPDs and 81 who did not (Fig. 1). Amongst analyzed patients, there were no deaths prior to discharge or within 30 postoperative days, for an STS operative mortality of 0 . PPDs were in place for a mean of 4.7 (st. dev 1.8) postoperative days. PPD placement was successful in all intended patients, and all PPDs worked appropriately up to removal. No direct complications of PPD placement were observed.

\section{Demographics and Operative Data}

No significant differences were identified between the PPD and non-PPD groups by sex, weight at surgery, preoperative creatinine, presence of premature birth ( $<36$ week gestation), presence of identified syndrome/chromosome abnormality, presence of low birth weight $(<2000 \mathrm{~g})$, and cross clamp time (Table 2). Significantly, PPD patients were younger at surgery, had longer bypass times, and were more likely to have delayed chest closure than non-PPD patients. The STAT-category distribution also significantly differed between groups, with PPD patients undergoing higher STAT-category procedures (Table 2). Inputs with $p$ values $\leq 0.1$ were included in multivariable analysis and include STAT category, bypass time, age at surgery, weight at surgery, and presence delayed chest closure (Table 2). Mean gestational age at birth was 38.3 (st dev

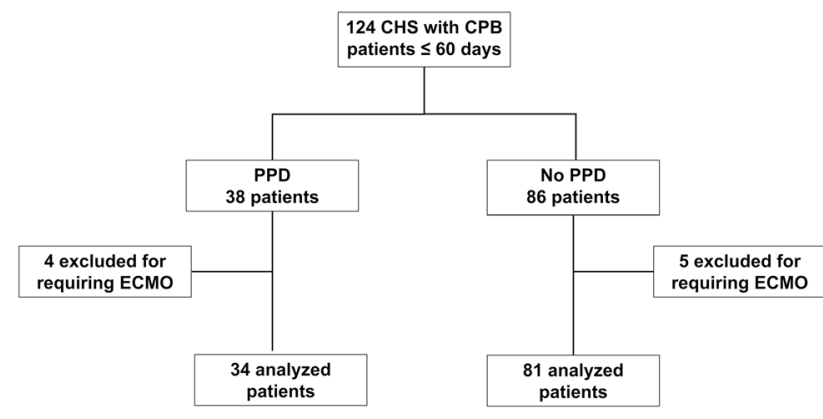

Fig. 1 Patient cohorts and exclusions. $C P B$ cardiopulmonary bypass, $C H S$ congenital heart surgery, ECMO extracorporeal membrane oxygenation, $P P D$ passive peritoneal drain
2.3) weeks for the PPD patients and 38.1 (st dev 2.6) weeks for the non-PPD patients.

\section{Vasoactive-Inotropic Scores}

PPD patients had significantly higher peak vasoactive-inotropic scores (VIS) on POD 0 in univariate analysis, but this was not seen in multivariable analysis. Peak daily VIS remained higher over each of POD 1-3 in both univariate and multivariable analysis (Table 3).

\section{AKI by KDIGO Creatinine Criteria}

Patients with a PPD had a significantly higher incidence of KDIGO Stage 1 AKI and KDIGO Stage 2 AKI by creatinine criteria in univariate analysis, although differences did not reach statistical significance after multivariable analysis (Table 3).

\section{Postoperative Hospital Length of Stay and Time to Chest Closure}

In univariate and multivariable analysis, there were no significant differences between PPD and non-PPD patients in total postoperative hospital length of stay (Table 4). All patients who emerged from the OR with an open chest later underwent chest closure. Of PPD patients that came from the OR with open chest (25 of 34), chest closure occurred at an average of 3.9 (st dev 0.9) postoperative days, while the average for non-PPD patients with delayed chest closure (18 of 81 ) was also 3.9 (st dev 0.9$)$ postoperative days $(p=0.824)$. Multivariable modeling for those with delayed chest closure demonstrated no significant difference between PPD and non-PPD patients in time to closure, with PPD presence accounting for $\mathrm{a}-0.3$ day difference in time to closure $(95 \%$ CI [-0.93, 0.32], $p=0.35$ ).

\section{Dynamic Respiratory Compliance and Postoperative Ventilator Hours}

In both the univariate and multivariable analyses, there was no significant difference between PPD and non-PPD patients in dynamic respiratory compliance at any time point examined (Table 4). In univariate analysis, postoperative hours of intubation were significantly higher in PPD patients, but a significant difference was not found after multivariable adjustment (Table 4).

\section{Postoperative Daily Weights and First Day Net Negative Fluid Balance}

In univariate and multivariable analysis, there was no significant difference between PPD and non-PPD groups in time 


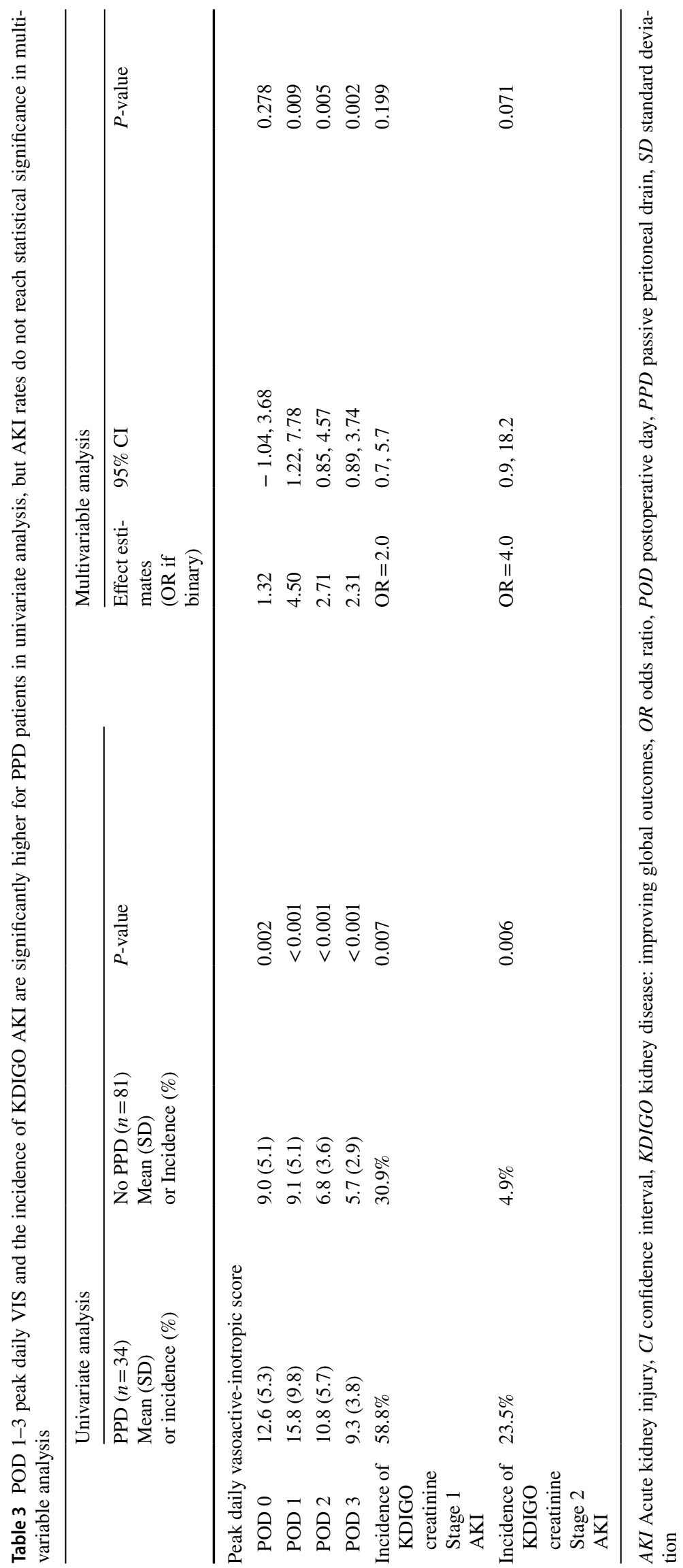




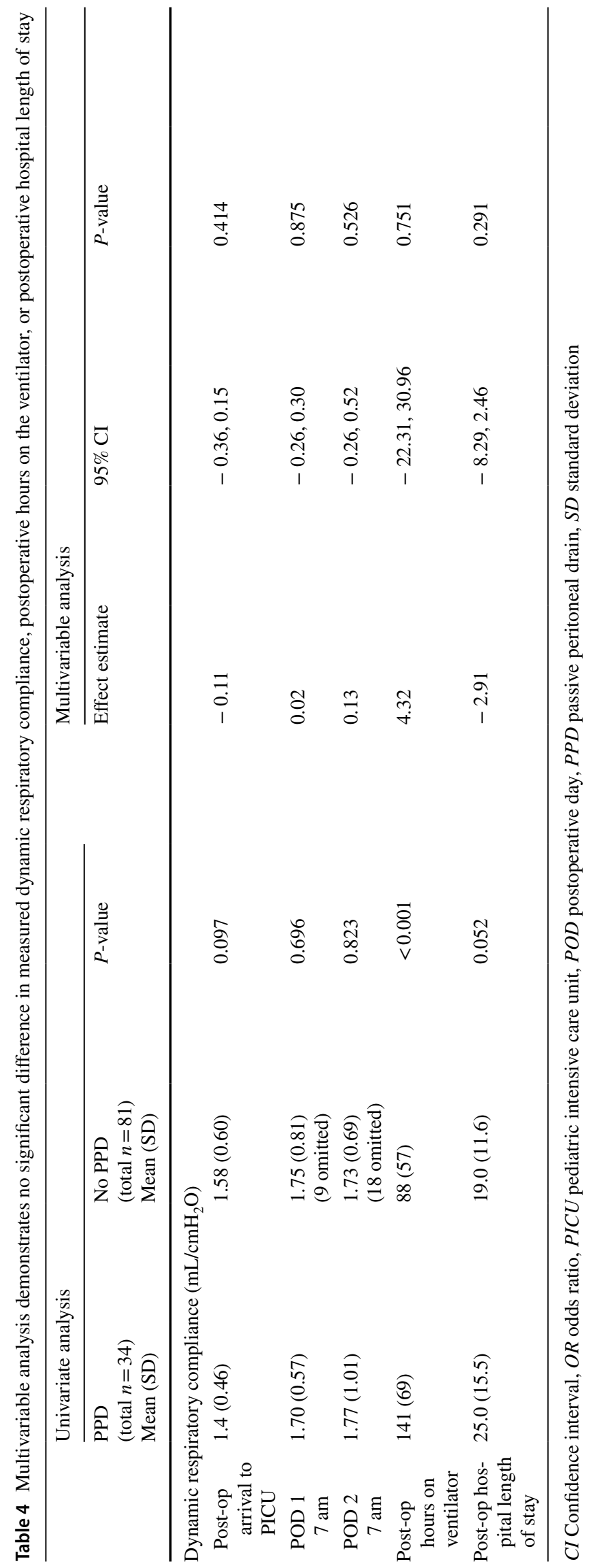


to first day with net negative fluid balance (Table 5). PPD patients had significantly higher percent weight increase on each of POD 1-5 in univariate analysis, but differences were not significant after multivariable adjustment (Table 5).

\section{Fluid Intake and Outputs}

In univariate and multivariable analyses, there were no significant differences in total fluid intake between PPD and non-PPD groups on POD 0 and POD 1-5 (Table 5). In univariate analysis, there was no significant difference in total fluid output between groups over POD 1-5. Cumulative urine output in PPD patients was significantly less over POD $1-5$ in univariate and multivariable analysis (effect $=-61.5$, 95\% CI [ $-110.3,-12.7], p=0.015)$. Use of PPD was associated with significantly higher total fluid output over POD $1-5$ in multivariable analysis (effect $=48.8,95 \%$ CI $[2.2$, 95.4], $\mathrm{p}=0.043$ ). PPDs produced a mean output of 107 (st dev 82) $\mathrm{mL} / \mathrm{kg}$ over POD 1-5 (Table 5).

\section{Diuretic Administration}

In univariate and multivariable analysis, PPD patients received significantly less furosemide than non-PPD patients over POD 1-5 (Table 5). In multivariable analysis, the presence of a PPD accounted for $3.41 \mathrm{mg} / \mathrm{kg}$ less over POD $1-5$ (95\% CI [1.69, 5.14], $p<0.001)$. Over POD $1-5$, one PPD patient received chlorothiazide and furosemide but the remaining PPD patients received no diuretics other than furosemide, while 15 non-PPD patients received chlorothiazide in addition to furosemide.

\section{Discussion}

In a cohort of neonates and young infants $<60$ days old who were non-randomly selected for greater preoperative and surgical risk, we demonstrate that empiric passive peritoneal drainage is associated with increased total fluid output over the first five postoperative days even in the setting of reduced exposure to diuretics and similar fluid input. The thirty-four patients who received PPDs tended to be higher acuity, as evidenced by significant differences in STAT categories, age at surgery, bypass time, and presence of delayed chest closure. We were unable to demonstrate benefit in endpoints such as duration of mechanical ventilation or hospital length of stay in this cohort of patients.

Even after multivariable adjustment for recognized possible confounders, maximum daily vasoactive-inotropic scores were higher in the PPD patients. It is possible that our multivariable model does not identify all confounders that make the PPD cohort higher risk and contribute to a higher maximum daily VIS. Notably, high VIS is associated with prolonged time to net negative fluid status in infants after cardiopulmonary bypass [12]. Our data demonstrates no significant difference in number of postoperative days to net negative despite the fact that PPD patients have significantly higher VIS.

Patients with PPD placement also had statistically higher incidences of KDIGO Stage 1 and Stage 2 acute kidney injury in univariate analysis, with a rate of $24 \%$ that is consistent with prior literature [13, 14]. Multivariable adjustment demonstrated no significant difference between the two groups for the incidence of KDIGO Stage $1 \mathrm{AKI}$, but the difference still trends toward significance for the incidence of KDIGO Stage 2 . We cannot entirely exclude the possibility that the presence of a PPD somehow drives the higher VIS scores and rate of stage $2 \mathrm{AKI}$ in these patients.

Recent work has demonstrated that fluid overload in neonatal patients after congenital heart surgery is associated with increased mortality and hospital length of stay independent of AKI, highlighting the importance of fluid management in these patients [1]. Kwiatkowski, et al., demonstrated shorter time to net negative fluid balance and improved VIS in a cohort of patients with peritoneal drains, $60 \%$ of whom received dialysis at some point. This work also showed enhanced interleukin 6 (IL-6) clearance and reduced duration of mechanical ventilation [4]. Other work has demonstrated that fluid removed via PPD or peritoneal dialysis contains pro-inflammatory IL-6 and IL-8 [15]. Work in an ischemic AKI mouse model has demonstrated that peritoneal dialysis clears IL-6 and reduces lung inflammation [16].

Our retrospective work examines the use of peritoneal drains with only passive drainage and does not demonstrate clear improvement in endpoints identified by Kwiatkowski, et al. [4]. It is possible that this is because PPD is not as effective at removal of inflammatory cytokines as dialysis. Relevant work by Sasser, et al., found that, in consecutive eras, chest tube and urine output were similar between PPD patients and peritoneal dialysis patients but that the dialysis patients had lower IL-6 and IL-8 concentrations at 24 postoperative hours [6].

Passive peritoneal drainage may be advantageous over peritoneal dialysis given its simplicity and that it may carry less risk of peritonitis. Saini, et al., demonstrated more rapid achievement of negative fluid balance in patients receiving a PPD after atrioventricular septal defect repairs [8]. In 22 patients undergoing the Norwood operation, those randomized to PPD had similar clinical outcomes to those without PPD, including hospital length of stay, time to lactate below $2 \mathrm{mmol}$ levels, time to extubation, and VIS [9]. Our broader patient cohort does not demonstrate significant difference between PPD and non-PPD groups in end-outcomes.

Kwiatkowski et al. showed a decrease in ventilator time for patients with a peritoneal catheter, whether used for 


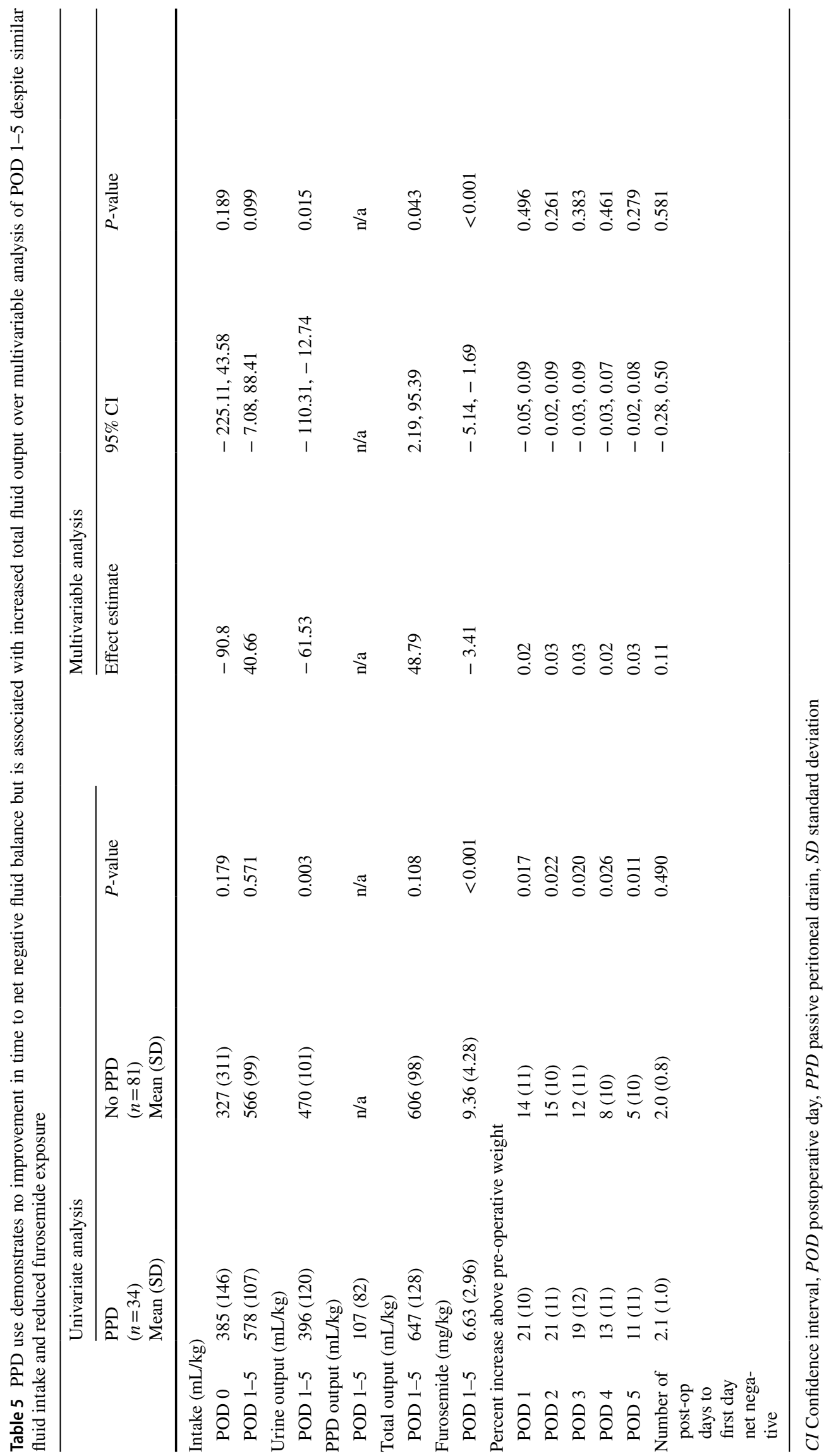


dialysis or passive drainage [4]. Our work found no significant difference in the number of postoperative ventilator hours for our PPD patients. Our practice is to remove the PPD prior to extubation in order to reduce the risk of omental herniation. It is possible that some patients remained on the ventilator longer while awaiting the removal of their PPD, which would explain our different finding. However, this seems unlikely, as our care teams favor removal of the PPD earlier than otherwise desired to facilitate extubation if the patient is ready.

In PPD patients, we typically hold, or give small doses of, diuretic until urine output exceeds PD drain output. This may account for the decreased diuretics given to PPD patients in our study. Although diuretic use is a necessary tool in the armamentarium of congenital heart surgery, it is not without some risks and side effects. Loop diuretic treatment is associated with fluid and electrolyte imbalances including hyponatremia, hypokalemia, and hypochloremic metabolic alkalosis, which can depress respiratory drive and delay ventilator weaning. Use of furosemide in preterm infants is also associated with nephrocalcinosis, which itself may lead to glomerular and tubular dysfunction [17].

In summary, we demonstrate that PPD placement enables greater total fluid output in the setting of less diuretic administration. Subjectively, prophylactic PPDs seem to provide an egress mechanism for third-spaced intracorporeal fluid during a time in which it is not otherwise mobilized due to factors including AKI, ongoing capillary leak, and subphysiologic oncotic pressures. Although our current study does not demonstrate endpoint benefit, we hypothesize that PPD may favorably alter fluid management in a subset of high-risk neonate and young infants as they recover from CHS. Specifically, we suspect that PPD may provide more benefit to patients expected to have significant postoperative diastolic dysfunction as opposed to those with complex operations and high risk of AKI but without expected significant diastolic dysfunction. Further studies should evaluate, in randomized fashion, the use of prophylactic passive peritoneal drainage for fluid management after CHS to determine which, if any, patients are most likely to benefit from this approach.

\section{Study Limitations}

Patient cohorts were broad and divergent in this modestsized retrospective study, and it is likely that our modeling does not account for all confounding preoperative and postoperative factors that may be associated with end outcomes. Patients were not randomized or blinded, and there were not predetermined study protocols for the selection of PPD patients or for the fluid management of either patient group. There was no specific protocol for timing of peritoneal drain removal. PPD patients had higher VIS scores and a trend towards higher rates of stage $2 \mathrm{AKI}$, and this retrospective work cannot entirely exclude the possibility that PPD presence somehow drives these findings. Treatment bias is a consideration, as it is possible that the decision to administer fluid resuscitation or vasoactive support could be influenced by the presence of a PPD. Analysis of electrolyte supplementation, especially in the setting of reduced diuretic administration, was not achievable given limitations in the data, wide array of postoperative critical illness, and variation in primary nutrition used in our patient cohorts.

\section{Conclusions}

PPD placement in a cohort of young infants undergoing CHS with higher surgical and preoperative risk enables improved total body postoperative fluid output even in the setting of reduced diuretic administration. In this comparison across broad and divergent groups of PPD and non-PPD patients, endpoint benefit was not demonstrated. PPD utilization in all infants after CHS is not necessary or advocated by this work, as it is likely that only certain groups of high-risk infants, if any, would benefit. Further evaluation and randomized controlled studies are warranted to determine which, if any, cohorts of high-risk patients would benefit from PPD.

Author Contributions All authors contributed to the study conception and design. Material preparation, data collection and analysis were performed by KP, NS, JD, and NM. The first draft of the manuscript was written by KP and all authors commented on previous versions of the manuscript. All authors read and approved the final manuscript.

Funding This research did not utilize funding.

Data Availability The authors are happy to make this available at request.

\section{Compliance with Ethical Standards}

Conflict of Interest The authors have no commercial or other associations that might pose a conflict of interest in connection with the submitted article. The authors of this work report no relationships with industry and other entities.

Ethics Approval This retrospective research was approved by the University of Wisconsin Institutional Review Board, protocol 20131598-CP009.

\section{References}

1. Mah KE, Hao S, Sutherland SM, Kwiatkowski DM, Axelrod DM, Almond CS, Krawczeski CD, Shin AY (2018) Fluid overload independent of acute kidney injury predicts poor outcomes in neonates following congenital heart surgery. Pediatr Nephrol 33(3):511-520. https://doi.org/10.1007/s00467-017-3818-x 
2. Kwiatkowski DM, Krawczeski CD (2017) Acute kidney injury and fluid overload in infants and children after cardiac surgery. Pediatr Nephrol 32(9):1509-1517. https://doi.org/10.1007/s0046 7-017-3643-2

3. Kwiatkowski DM, Goldstein SL, Cooper DS, Nelson DP, Morales DL, Krawczeski CD (2017) Peritoneal dialysis vs furosemide for prevention of fluid overload in infants after cardiac surgery: a randomized clinical trial. JAMA Pediatr 171(4):357-364. https ://doi.org/10.1001/jamapediatrics.2016.4538

4. Kwiatkowski DM, Menon S, Krawczeski CD, Goldstein SL, Morales DL, Phillips A, Manning PB, Eghtesady P, Wang Y, Nelson DP, Cooper DS (2015) Improved outcomes with peritoneal dialysis catheter placement after cardiopulmonary bypass in infants. J Thorac Cardiovasc Surg 149(1):230-236. https://doi. org/10.1016/j.jtcvs.2013.11.040

5. Madenci AL, Thiagarajan RR, Stoffan AP, Emani SM, Rajagopal SK, Weldon CB (2013) Characterizing peritoneal dialysis catheter use in pediatric patients after cardiac surgery. $\mathrm{J}$ Thorac Cardiovasc Surg 146(2):334-338. https://doi.org/10.1016/j.jtcvs.2012.10.009

6. Sasser WC, Dabal RJ, Askenazi DJ, Borasino S, Moellinger AB, Kirklin JK, Alten JA (2014) Prophylactic peritoneal dialysis following cardiopulmonary bypass in children is associated with decreased inflammation and improved clinical outcomes. Congenit Heart Dis 9(2):106-115. https://doi.org/10.1111/chd.12072

7. Averbuch N, Birk E, Frenkel G, Gogia O, Shulman OM, Bruckheimer E, Nachum E, Amir G (2014) Percutaneous intraperitoneal catheters in neonates following open heart surgery. J Intensive Care Med 29(3):160-164. https://doi.org/10.1177/0885066613 482086

8. Saini A, Delius RE, Seshadri S, Walters H, Mastropietro CW (2012) Passive peritoneal drainage improves fluid balance after surgery for congenital heart disease. Eur J Cardiothorac Surg 41(2):256-260. https://doi.org/10.1016/j.ejcts.2011.05.037

9. Ryerson LM, Mackie AS, Atallah J, Joffe AR, Rebeyka IM, Ross DB, Adatia I (2015) Prophylactic peritoneal dialysis catheter does not decrease time to achieve a negative fluid balance after the Norwood procedure: a randomized controlled trial. J Thorac Cardiovasc Surg 149(1):222-228. https://doi.org/10.1016/j.jtcvs .2014.08.011

10. Jetton JG, Boohaker LJ, Sethi SK, Wazir S, Rohatgi S, Soranno DE, Chishti AS, Woroniecki R, Mammen C, Swanson JR, Sridhar S, Wong CS, Kupferman JC, Griffin RL, Askenazi DJ, Neonatal
Kidney C (2017) Incidence and outcomes of neonatal acute kidney injury (AWAKEN): a multicentre, multinational, observational cohort study. Lancet Child Adolesc Health 1(3):184-194. https:// doi.org/10.1016/S2352-4642(17)30069-X

11. Selewski DT, Charlton JR, Jetton JG, Guillet R, Mhanna MJ, Askenazi DJ, Kent AL (2015) Neonatal acute kidney injury. Pediatrics 136(2):e463-473. https://doi.org/10.1542/peds.2014-3819

12. Gaies MG, Gurney JG, Yen AH, Napoli ML, Gajarski RJ, Ohye RG, Charpie JR, Hirsch JC (2010) Vasoactive-inotropic score as a predictor of morbidity and mortality in infants after cardiopulmonary bypass. Pediatr Crit Care Med 11(2):234-238. https://doi. org/10.1097/PCC.0b013e3181b806fc

13. Ueno K, Seki S, Shiokawa N, Matsuba T, Miyazono A, Hazeki D, Imoto Y, Kawano Y (2019) Validation of acute kidney injury according to the modified KDIGO criteria in infants after cardiac surgery for congenital heart disease. Nephrology (Carlton) 24(3):294-300. https://doi.org/10.1111/nep.13240

14. Ueno K, Shiokawa N, Takahashi Y, Nakae K, Kawamura J, Imoto Y, Kawano Y (2019) Kidney disease: improving global outcomes in neonates with acute kidney injury after cardiac surgery. Clin Exp Nephrol 24(2):167-173. https://doi.org/10.1007/s10157-01901805-7

15. Barhight MF, Soranno D, Faubel S, Gist KM (2018) Fluid management with peritoneal dialysis after pediatric cardiac surgery. World J Pediatr Congenit Heart Surg 9(6):696-704. https://doi. org/10.1177/2150135118800699

16. Altmann C, Ahuja N, Kiekhaefer CM, Andres Hernando A, Okamura K, Bhargava R, Duplantis J, Kirkbride-Romeo LA, Huckles J, Fox BM, Kahn K, Soranno D, Gil HW, Teitelbaum I, Faubel S (2017) Early peritoneal dialysis reduces lung inflammation in mice with ischemic acute kidney injury. Kidney Int 92(2):365376. https://doi.org/10.1016/j.kint.2017.01.020

17. Pacifici GM (2013) Clinical pharmacology of furosemide in neonates: a review. Pharmaceuticals 6(9):1094-1129. https://doi. org/10.3390/ph6091094

Publisher's Note Springer Nature remains neutral with regard to jurisdictional claims in published maps and institutional affiliations. 